\title{
Signaling from Integrins to Fyn to Rho Family GTPases Regulates Morphologic Differentiation of Oligodendrocytes
}

\author{
Xiquan Liang, ${ }^{1}$ Nicole A. Draghi, ${ }^{2}$ and Marilyn D. Resh ${ }^{1,2}$ \\ ${ }^{1}$ Cell Biology Program, Memorial Sloan-Kettering Cancer Center, and ${ }^{2}$ Graduate Program in Biochemistry and Structural Biology, Weill Graduate School of \\ Medical Sciences of Cornell University, New York, New York 10021
}

\begin{abstract}
Differentiation of oligodendrocyte progenitor cells requires activation of the Src family kinase Fyn. The signals that are upstream and downstream of Fyn in oligodendrocytes remain essentially unknown. Here we show that extracellular matrix engagement regulates the morphology of oligodendrocytes and activates Fyn. Infection of primary oligodendrocyte cultures with recombinant adenovirus revealed that expression of Fyn or its downstream target p190RhoGAP induced process extension. This phenotypic change was not observed when kinase-inactive Fyn or GAP-defective p190 mutants were expressed. Because Rho family proteins are regulated by p190, we monitored the effects of introducing dominant-negative (DN) or constitutively activated (CA) versions of Rho, Racl, or Cdc42 into primary oligodendrocyte cultures. Expression of DN Rho, CA Rac1, or CA Cdc42 induced outgrowth of oligodendrocyte processes, whereas introduction of CA Rho, DN Rac1, or DN cdc42 inhibited oligodendrocyte differentiation, indicating that Rho and Cdc42-Racl exert opposing effects on oligodendrocyte differentiation. Direct measurement of Rho family activity revealed that RhoA was downregulated, and Cdc42 and Racl were upregulated during differentiation of primary oligodendrocytes. Moreover, inhibition of integrin engagement or of Fyn activation blocked activation of Rac1 and cdc42 as well as myelin basic protein expression. Taken together, these results suggest a linear signal transduction pathway of integrin-Fyn-Rho family GTPases that controls morphologic differentiation of oligodendrocytes.
\end{abstract}

Key words: kinase; oligodendrocyte; RhoA; Fyn; signaling; myelination; integrins

\section{Introduction}

Myelin formation in the CNS occurs when oligodendrocyte precursor cells (OPCs) proliferate, migrate throughout the CNS, and differentiate into mature oligodendrocytes. The Src family kinase Fyn plays an important role in oligodendrocyte differentiation. In Fyn $-/-$ mice, the myelin sheath is thinner and more irregular than in wild-type (wt) mice. The content of myelin basic protein (MBP) in Fyn $-/-$ mice is $\sim 50 \%$ of that in wild-type mice, and transactivation of the MBP gene by Fyn regulates myelination (Umemori et al., 1999; Sperber et al., 2001). Moreover, Fyn is the only Src family kinase that is upregulated during oligodendrocyte differentiation, and treatment of oligodendrocytes with Src family kinase inhibitors blocks oligodendrocyte differentiation (Osterhout et al., 1999).

One potential mechanism to activate Fyn is via integrin engagement. Oligodendrocytes express integrins $\alpha 6 \beta 1, \alpha v \beta 1$, $\alpha \mathrm{v} \beta 3$, and $\alpha \mathrm{v} \beta 5$ (Milner et al., 1994). Integrin expression is regulated during differentiation, with expression of $\alpha \mathrm{v} \beta 1$ downregulated and $\alpha \mathrm{v} \beta 5$ upregulated (Milner and ffrench-Constant, 1994). Proliferation and differentiation of OPCs are regulated by

Received Dec. 2, 2003; revised June 30, 2004; accepted July 1, 2004.

This work was supported by Grant RG-3235-A-2 from the National Multiple Sclerosis Society and Grant CA96582 from the National Institutes of Health. We thank Drs. Moses Chao, Hiroko Yano, and Rebecca Wolf for technical advice, Dr. Sue Barnett for the 02AMyc cells, Dr. Keith Burridge for GST-RBD and GST-PBD constructs, the Guthrie CDNA Resource Center for Rho family CDNAs, and Dr. Filippo G. Giancotti for a critical reading of this manuscript.

Correspondence should be addressed to Dr. Marilyn D. Resh, Cell Biology Program, Memorial Sloan-Kettering Cancer Center, 1275 York Avenue, Box 143, New York, NY 10021. E-mail: m-resh@ski.mskcc.org.

DOI:10.1523/JNEUROSCI.5319-03.2004

Copyright $\odot 2004$ Society for Neuroscience $\quad$ 0270-6474/04/247140-10\$15.00/0 $\alpha \mathrm{v}$ integrins (Blaschuk et al., 2000). Disruption of oligodendrocyte adhesion to a glial-derived matrix decreases MBP and proteolipid protein mRNA levels (Malek-Hedayat and Rome, 1994), suggesting that integrin signaling induces myelin gene expression and oligodendrocyte differentiation.

Integrin engagement triggers multiple signaling cascades, including activation of tyrosine kinases and Rho family GTPases (Price et al., 1998; Ren et al., 1999; del Pozo et al., 2000; Arthur and Burridge, 2001; Berrier et al., 2002). Rho family GTPases act as molecular switches by cycling between GTP-bound (on) and GDP-bound (off) states. Guanine nucleotide exchange factors (GEFs) stimulate exchange of GDP for GTP to generate the active form, whereas GTPase-activating proteins (GAPs) accelerate the intrinsic GTPase activity to inactivate the switch. Several mechanisms link integrin engagement with activation of the Rho family protein Rac, including signaling by focal adhesion kinase (Schlaepfer et al., 1997; Schaller et al., 1999; Das et al., 2000), p130Cas (Klemke et al., 1998; Cheresh et al., 1999), and Vav family GEFs (Schwartz, 2001).

Rho family GTPases play an essential role in the control of cellular morphology. In fibroblasts, Rho activation promotes stress fiber formation, whereas activation of Cdc42 and Rac induces lamellipodia and filopodia, respectively (Ridley et al., 1992; Kozma et al., 1995; Nobes and Hall, 1995). It is likely that Rho, Cdc42, and/or Rac regulate the cell shape changes associated with oligodendrocyte differentiation. Indeed, our laboratory demonstrated that overexpression of dominant-negative RhoA causes hyperextension of oligodendrocyte processes (Wolf et al., 2001). 
We set out to identify the upstream regulators and downstream effectors of Fyn and study their function in oligodendrocyte differentiation. Here we show that integrin engagement activates Fyn. Fyn phosphorylates and activates p190RhoGAP, which in turn inactivates Rho and causes alterations in oligodendrocyte morphology. In addition, Fyn activates Cdc42 and Rac, which also triggers morphologic differentiation of oligodendrocytes. Thus, Rho and Cdc42-Rac exhibit opposing effects on oligodendrocyte morphologic complexity. These results provide evidence for a linear signal transduction pathway of integrinFyn-Rho family GTPases that regulates morphologic differentiation of oligodendrocytes.

\section{Materials and Methods}

Materials. Poly-D-lysine (PDL), bovine fibronectin (FN), vitronectin, insulin, transferrin, progesterone, putrescine, and sodium selenite were purchased from Sigma-Aldrich (St. Louis, MO). PP3 and PP2 were obtained from Calbiochem (La Jolla, CA). Laminin-2 (merosin) was purchased from Invitrogen (Carlsbad, CA). Recombinant human PDGF-AA and bFGF were purchased from PeproTech Inc. (Rocky Hill, NJ). The following antibodies were used for immunoprecipitation, Western blotting, or immunofluorescence: mouse anti-HA (Santa Cruz Biotechnology, Santa Cruz, CA), mouse anti-RhoA, rabbit Cdc42 (Santa Cruz Biotechnology), mouse anti-MBP (Chemicon, Temecula, CA), mouse antiRac, mouse anti-Fak (Upstate Biotechnology, Lake Placid, NY), mouse anti-Fyn, and hamster anti-rat CD29 (integrin $\beta-1$ ) (BD Biosciences, Franklin Lakes, NJ) antibodies. The rabbit polyclonal antiserum to Fyn used for immunoprecipitation was described previously (van't Hof and Resh, 1997).

Plasmids. pAdeasy-1 and pShuttle-CMV were generously provided by Dr. James R. Bamburg (Colorado State University, Fort Collins, CO). pFB-neo and pShuttle-IRES-hrGFP-2 were purchased from Stratagene (La Jolla, CA). pEGFP-N1 was purchased from Clontech. The EGFP insert was subcloned into pShuttle-CMV at the BglII-NotI site. Fulllength HA-tagged wild-type p190RhoGAP and GAP-defective (R1283A)p190RhoGAP mutants were prepared as described (Wolf et al., 2001). To clone wild-type and mutants of p190RhoGAP into pShuttle vectors, p190 was first inserted into pFB-neo vectors at EcoRI sites using PCR and was then subcloned into pShuttle vectors at Sal-NotI sites.

To prepare full-length FynEGFP fusion constructs, PCR products of wild-type, G2A, or K299M Fyn mutant with the stop codon deleted were inserted into pEGFP-N1 at EcoRI-BamHI sites. The fragment containing FynEGFP was subcloned into pFB-neo at EcoRI-NotI sites. Finally, the FynEGFP fragment was subcloned into pShuttle-CMV at Sal-NotI sites.

$\mathrm{N}$-terminal $3 \times$ HA-tagged CDC42(G12V), CDC42(T17N), RhoA(G14V), RhoA(T19N), Rac1(G12V), and $\operatorname{Racl}(\mathrm{T} 17 \mathrm{~N})$ in pcDNA3.1 were generously provided by the Guthrie cDNA Resource Center (Sayre, PA). Rac1(G12V), and Rac1(T17N) were subcloned into pShuttle-IRES-hrGFP at the NheI and XhoI sites. RhoA(G14V) and RhoA(T19N) were subcloned into pShuttle-CMV at the KpnI and XhoI sites and then subcloned into pShuttle-IRES-hrGFP at the BglII and XhoI sites. CDC42(G12V) and CDC42(T17N) were subcloned into pShuttleIRES-hrGFP at the NotI and SalI sites using PCR. The shuttle vectors were linearized with PmeI and were cotransformed with the pAdeasy-1 vector into BJ5183 cells (Stratagene) to generate recombinant Adeasy plasmid DNA.

Cell culture. Isolation of oligodendrocyte precursor cells (OPCs) was previously described (Wolf et al., 2001). Briefly, primary oligodendrocyte precursors were grown for $\sim 9 \mathrm{~d}$ in DMEM medium supplemented with $15 \% \mathrm{FBS}$ at $37^{\circ} \mathrm{C}$ and $5 \% \mathrm{CO}_{2}$. Flasks were preshaken at $300 \mathrm{rpm}$ for $10 \mathrm{~min}$ to remove microglia from the culture, replaced with fresh medium, and incubated for $5-6 \mathrm{hr}$ at $37^{\circ} \mathrm{C}$ and $5 \% \mathrm{CO}_{2}$ before being shaken at $190 \mathrm{rpm}$ overnight to dislodge the loosely attached oligodendrocyte precursors. OPCs were further purified from astrocytes by plating them in uncoated tissue culture dishes for $\sim 40 \mathrm{~min}$. More than $80 \%$ of the isolated OPCs were A2B5-positive (data not shown). Purified OPCs were cultured on PDL- or FN-coated dishes for 6-16 hr. Cells were lysed or induced to differentiate for $4-6 \mathrm{~d}$ by switching to a defined differentia- tion DMEM medium [high glucose, $0.5 \%$ FBS, $50 \mu \mathrm{g} / \mathrm{ml}$ human apotransferrin (Sigma), $30 \mathrm{~nm}$ sodium selenite, $30 \mathrm{~nm}$ 3,3,5 tri-iodo-Lthryronine (Sigma), and $50 \mathrm{ng} / \mathrm{ml}$ insulin]. For biochemical studies, differentiated cells were lysed in radioimmunoprecipitation assay buffer containing $50 \mathrm{~mm}$ Tris- $\mathrm{HCl}$, pH 8.0, $150 \mathrm{~mm} \mathrm{NaCl}, 5 \mathrm{~mm}$ EDTA, $1 \%$ NP-40, $0.5 \%$ sodium deoxycholate, $0.1 \%$ SDS, $10 \mathrm{~mm} \mathrm{NaF}, 1 \mathrm{~mm}$ $\mathrm{Na}_{3} \mathrm{VO}_{4}, 0.5 \mathrm{~mm}$ PMSF, $0.5 \mu \mathrm{g} / \mathrm{ml}$ leupeptin, and $0.5 \mu \mathrm{g} / \mathrm{ml}$ aprotinin. To expand oligodendrocyte progenitor cells, cells were cultured in OPC proliferation DMEM medium containing $5 \mu \mathrm{g} / \mathrm{ml}$ insulin, $100 \mu \mathrm{g} / \mathrm{ml}$ transferrin, $20 \mathrm{~nm}$ progesterone, $100 \mu \mathrm{M}$ putrescine, $30 \mathrm{~nm}$ sodium selenite, $10 \mathrm{ng} / \mathrm{ml}$ PDGF-AA, and $10 \mathrm{ng} / \mathrm{ml} \mathrm{bFGF}$.

The O2A myc oligodendrocyte cell line (Barnett and Crouch, 1995) was grown on PDL-coated dishes in DMEM high glucose supplemented with $4.5 \mathrm{gm} / \mathrm{l}$ glucose, $1 \mathrm{~mm}$ pyruvate, $2 \mathrm{~mm}$ L-glutamine, $100 \mu \mathrm{g} / \mathrm{ml}$ penicillin-streptomycin, $0.1 \mathrm{mg} / \mathrm{ml}$ bovine serum albumin, $60 \mu \mathrm{g} / \mathrm{ml}$ $N$-acetyl-L-cysteine, $100 \mu \mathrm{M}$ putrecine, $5 \mu \mathrm{g} / \mathrm{ml}$ insulin, $6.28 \mathrm{ng} / \mathrm{ml}$ progesterone, $100 \mu \mathrm{g} / \mathrm{ml}$ transferrin, $5.2 \mathrm{ng} / \mathrm{ml}$ sodium selenite, $20 \mathrm{ng} / \mathrm{ml}$ bFGF and $10 \mathrm{ng} / \mathrm{ml}$ PDGF. For differentiation, growth factors were removed, and the medium was supplemented with $20.2 \mathrm{ng} / \mathrm{ml}$ tri-iodo-thyronine.

Adenovirus preparation and infection. To generate adenovirus, recombinant Adeasy plasmid DNA ( $3 \mu \mathrm{g})$ was linearized with PacI and transfected into a $60 \mathrm{~mm}$ plate of 293 cells using Lipofectamine 2000 (Invitrogen). The progress of infection was monitored by green fluorescence microscopy. Cells were cultured 7-10 d, and primary viral stock was prepared with four rounds of freezing and thawing. Amplification of a primary viral stock was achieved by infection of fresh 293 cells. For adenoviral infection of oligodendrocytes, freshly isolated progenitors were incubated with diluted adenoviral stocks (10-50 multiplicities of infection) overnight in DMEM medium supplemented with 15\% FBS or in DMEM proliferation medium. Progenitors were then induced to differentiate for $2 \mathrm{~d}$ by changing to the defined differentiation medium.

Immune complex kinase assays. Cell lysates from progenitors or differentiated oligodendrocytes were immunoprecipitated with polyclonal anti-Fyn antibody. Immune complexes were washed with lysis buffer and incubated with $30 \mu \mathrm{l}$ of kinase buffer containing $20 \mathrm{~mm}$ Tris- $\mathrm{HCl}, \mathrm{pH} 7.4$, $150 \mathrm{~mm} \mathrm{NaCl}, 10 \mathrm{mM} \mathrm{MnCl}_{2}, 10 \mathrm{mM} \mathrm{MgCl}_{2}$, and $10 \mu \mathrm{Ci}$ of $\left[\gamma^{-32} \mathrm{P}\right] \mathrm{ATP}$ at $30^{\circ} \mathrm{C}$ for $20 \mathrm{~min}$. Reactions were terminated by the addition of SDS sample buffer. The $\left[\gamma^{32} \mathrm{P}\right]$-labeled proteins were analyzed by phosphorimaging.

Rho family GTPase activities. GST-Rhotekin-binding domain (RBD) and GST-PAK binding domain (PBD) were kindly provided by Dr. Keith Burridge (University of North Carolina, Chapel Hill, NC). Small GTPase activities were measured similarly as described (Ren et al., 1999). Briefly, progenitor and differentiated oligodendrocytes were lysed in $300 \mu \mathrm{l}$ of 50 mu Tris, $\mathrm{pH} 7.4$, containing $1 \%$ Triton $\mathrm{X}-100,0.1 \%$ SDS, $0.5 \%$ deoxycholate, $500 \mathrm{~mm} \mathrm{NaCl}$, and $0.5 \mathrm{~mm} \mathrm{MgCl}_{2}$. Cell lysates $(200-500 \mu \mathrm{g})$ were clarified at $100,000 \times g$ for $15 \mathrm{~min}$ and incubated for $40 \mathrm{~min}$ with 20 $\mu \mathrm{g}$ of GST fusion proteins containing the Rhotekin-binding domain (for RhoA assay) or the PAK-binding domain (for Racl and cdc42 assays) bound to glutathione-Sepharose beads (Amersham Biosciences, Uppsala, Sweden). Samples were washed with lysis buffer and then immunoblotted with anti-RhoA, Rac1, or cdc42 antibodies. Whole-cell lysates were directly immunoblotted to determine the total amount of Rho, Rac, or Cdc42 proteins. To determine the effect of anti- $\beta 1$ antibody on Cdc42-Rac activities, oligodendrocyte progenitor cells were preincubated with control serum or anti- $\beta 1$ antibody $(20 \mu \mathrm{g} / \mathrm{ml})$ for $1 \mathrm{hr}$ in differentiation medium and were then plated in differentiation medium in the presence of control serum or anti-integrin $\beta 1$ antibody on $\mathrm{FN}$ coated dishes for $5 \mathrm{~d}$. Activities of Cdc42-Rac were measured as described above. To determine the effect of Src family kinase inhibitor on Cdc42-Rac activities, oligodendrocyte progenitor cells were induced to differentiate for $2 \mathrm{~d}$ in the presence of $3 \mu \mathrm{M}$ PP2 or the nonfunctional analog PP3, and activities of Cdc42-Rac were determined.

Immunofluorescence staining. Purified OPCs were plated onto PDLcoated glass coverslips, cultured for $\sim 4-6 \mathrm{hr}$, and then were infected overnight with various adenoviruses. The progenitor cells were washed with PBS and cultured in a defined differentiation medium for $2 \mathrm{~d}$ and then examined live. Alternatively, cells were fixed in $3.7 \%$ paraformaldehyde for $15 \mathrm{~min}$, washed with PBS, and then permeabilized with $0.2 \%$ 
(A)
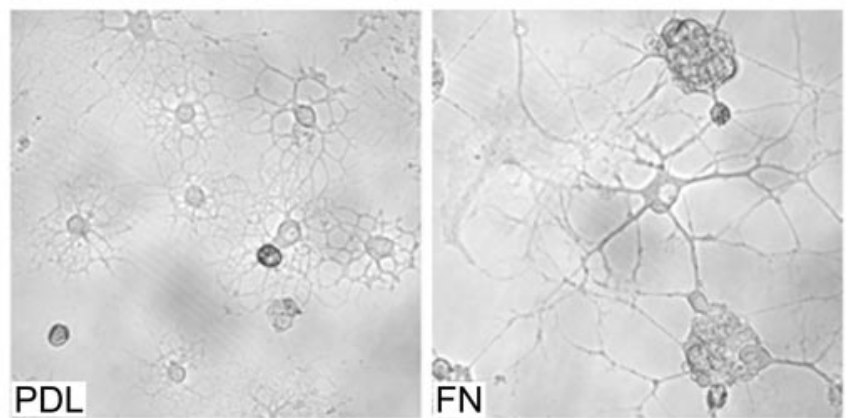

(B)

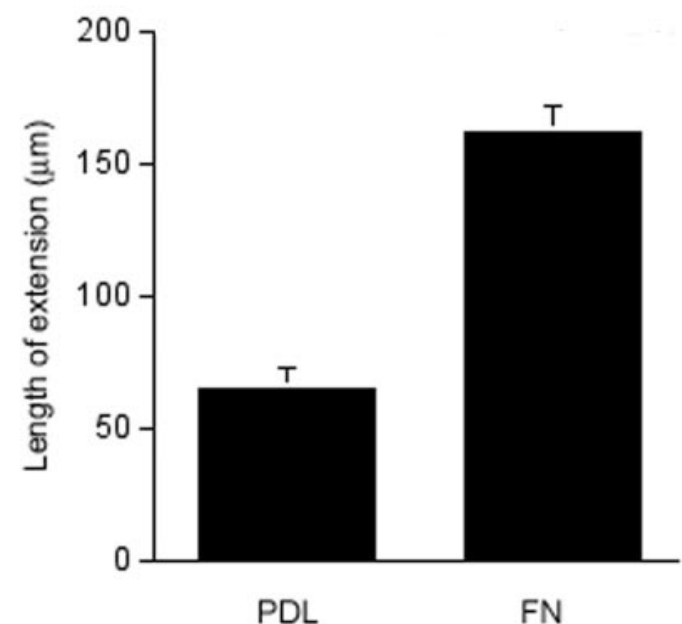

(C)
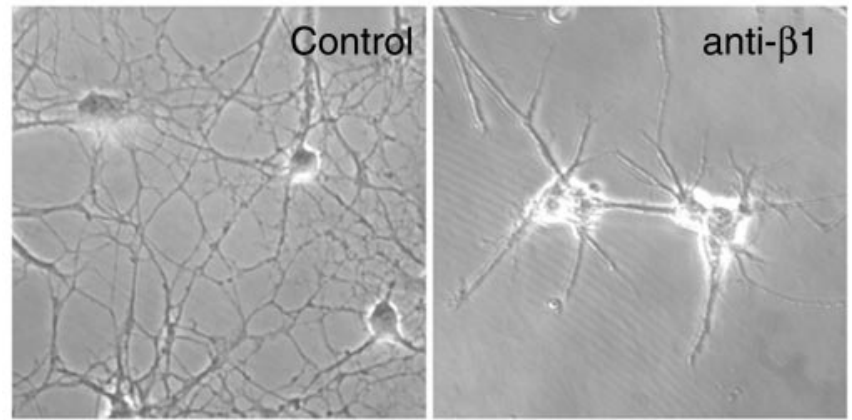

Figure 1. Effect of extracellular matrix on oligodendrocyte differentiation. $A, O P C s$ plated on PDL-coated or FN-coated dishes were induced to differentiate for 5 d in differentiation medium. The morphology of the cells was visualized by phase-contrast microscopy. B, Quantitation of the lengths of processes using MetaMorph Imaging. C, Freshly isolated OPCs were preincubated with control serum or anti- $\beta 1$ antibody $(20 \mu \mathrm{g} / \mathrm{ml})$ for $1 \mathrm{hr}$ in differentiation medium and were then plated on $\mathrm{FN}$-coated dishes for $5 \mathrm{~d}$ in the presence of control serum or anti-integrin $\beta 1$ antibody. Cell morphology was examined using phase-contrast microscopy.

Triton X-100 in PBS for 5 min. After washing with PBS, cells were incubated with 4\% normal donkey serum (Jackson ImmunoResearch, West Grove, PA) in PBS for $1 \mathrm{hr}$ to block nonspecific binding sites, and then incubated for $1.5 \mathrm{hr}$ with primary antibodies in $4 \%$ donkey serum. After extensive washing with PBS, cells were incubated for $1 \mathrm{hr}$ with Alexa Red
(A)

\section{[ $\left.{ }^{32} \mathrm{P}\right]$-labeled Fyn}

P D

C anti- $\beta 1$

WB: anti-Fyn

WB: anti-Fak
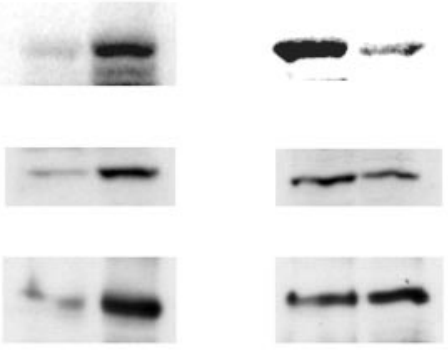

(B)
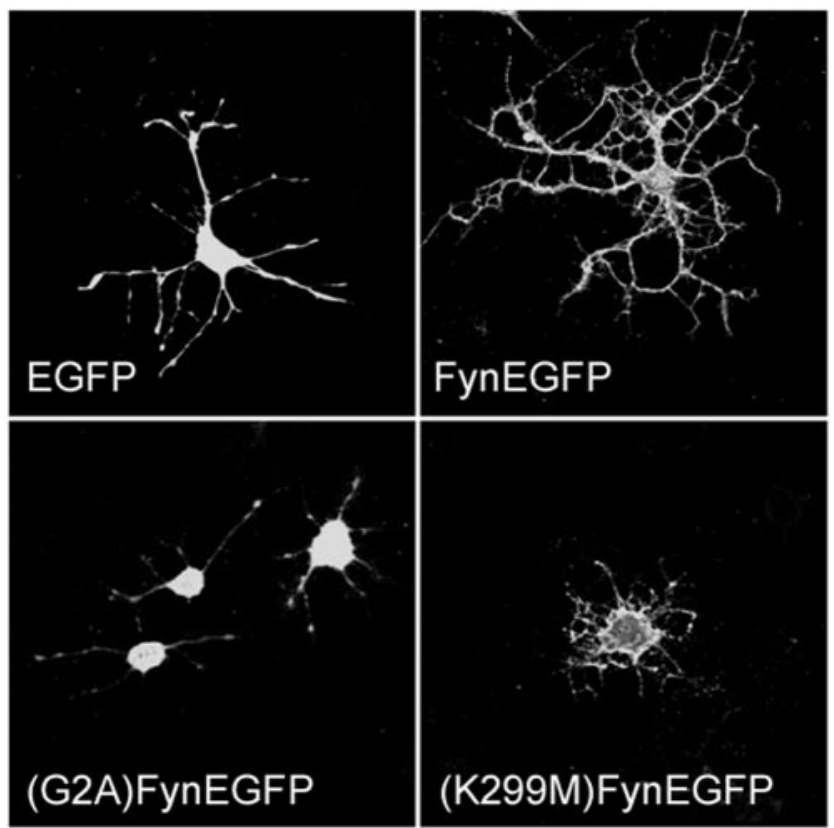

Figure 2. Fyn is regulated by integrins and is required for OPC differentiation. $A$, Oligodendrocyte progenitor cells $(P)$ grown on $\mathrm{FN}$-coated dishes were induced to differentiate for 5 dinto mature oligodendrocytes (D). Cell lysates were immunoprecipitated using anti-Fyn antibody, and immunocomplexes were assayed for autophosphorylation activities in the presence of [ $\gamma^{-}{ }^{32}$ P]ATP. To examine the effect of anti-integrin $\beta 1$ antibody on Fyn activation, OPCs were preincubated with control serum or anti-integrin $\beta 1$ antibody and then induced to differentiate for $5 \mathrm{~d}$ in the presence of control serum or anti-integrin $\beta 1$ antibody. B, OPCs were infected overnight with adenoviruses expressing EGFP, FynEGFP, (G2A)FynEGFP, or (K299M)FynEGFP and were then induced to differentiate for $2 \mathrm{~d}$ in defined differentiation medium. Live cells grown on PDL-coated coverslips were examined using confocal microscopy. Pictures shown are representative of five independent experiments.

conjugated goat anti-mouse secondary antibody (Molecular Probes, Eugene, OR) at a dilution of 1:500 in 4\% donkey serum. After extensive washing with PBS, coverslips were mounted on slides using Prolong Antifade mounting media (Molecular Probes). The cells were viewed using a Zeiss (Oberkochen, Germany) LSM510 confocal microscope.

\section{Results}

Effect of extracellular matrix on OPC morphologic differentiation

To examine the role of extracellular matrix-integrin interactions in oligodendrocyte differentiation, OPCs were freshly isolated from neonatal rat brain and were plated on either PDL- or FNcoated dishes for 6-16 hr. Freshly isolated OPCs did not adhere 
well to merosin-coated dishes (data not shown). OPCs were then induced to differentiate in differentiation medium for 4-6 d, at which point they became O4positive (data not shown). Process extension was examined by phase contrast microscopy, and the length of extended processes was measured using the MetaMorph analytic program. Obvious morphological differences were observed between the cells plated on PDL-coated and FN-coated dishes (Fig. 1A). Although the processes of oligodendrocytes plated on PDL were more highly branched than those plated on FN, process extension length was almost threefold greater when cells were plated on FN compared with PDL (Fig. 1B). The morphology of oligodendrocytes plated on vitronectin was similar to that of cells plated on FN (data not shown). These data suggest that extracellular matrix regulates the morphology of oligodendrocytes. To address the function of integrins on OPC differentiation, freshly isolated OPCs were preincubated with control serum or anti-integrin $\beta 1$ antibody in differentiation medium for $1 \mathrm{hr}$, and then plated on FN-coated dishes. The cells were allowed to differentiate in the presence of control serum or anti-integrin $\beta 1$ antibody for $5 \mathrm{~d}$, and their morphology was examined by phase-contrast microscopy. As depicted in Figure $1 C$, process extension of OPC was significantly inhibited in the presence of anti-integrin $\beta 1$ antibody. These findings suggest that integrins regulate OPC morphologic differentiation and are consistent with the observation that integrin $\beta 1$ is essential for myelination in vivo (Relvas et al., 2001).

\section{Fyn is regulated by integrins and is required for $O P C$ morphologic differentiation}

We previously showed that upregulation of the Src family kinase Fyn is required for

OPC differentiation (Osterhout et al., 1999). Next, we tested whether integrins were responsible for Fyn activation. Freshly isolated OPCs were preincubated with control serum or antiintegrin $\beta 1$ antibody and then induced to differentiate for $4-6 \mathrm{~d}$ on FN-coated dishes in the presence of control serum or antiintegrin $\beta 1$ blocking antibody. Cells were lysed and immunoprecipitated with anti-Fyn antibody, and immunocomplexes were assayed for Fyn autophosphorylation in the presence of $\left[\gamma^{-}{ }^{32} \mathrm{P}\right]$ ATP. The expression level of Fyn was determined by Western blotting using anti-Fyn antibody. As depicted in Figure 2 , both the kinase activity and expression level of Fyn were upregulated during oligodendrocyte differentiation, consistent with our previous findings (Osterhout et al., 1999). Treatment of cells with anti-integrin $\beta 1$ blocking antibody blocked Fyn upregulation, suggesting that integrins regulate the activation of Fyn during oligodendrocyte differentiation. To ensure that the blocking antibody was not inducing general cell toxicity, we examined the levels of Fak, another protein that we have observed to be regulated during OPC differentiation. As depicted in Figure 2, no change in the levels of Fak was observed when cells were treated with anti-integrin $\beta 1$ antibody.

Our previous studies showed that treatment of oligodendrocytes with Src family kinase inhibitors blocked differentiation of OPCs. We next examined whether direct introduction of Fyn would induce alterations in oligodendrocyte morphology. Because it is extremely difficult to transfect OPCs efficiently, we generated adenoviruses expressing full-length EGFP fusions of wild-type Fyn, (G2A)Fyn, or kinase-dead (K299M)Fyn mutants. Approximately $80 \%$ of the cells became infected with the recombinant adenoviruses. (G2A)Fyn is a Fyn mutant that is neither myristoylated nor palmitoylated and cannot bind membranes (Liang et al., 2001). OPCs were infected overnight with recombinant adenovirus in the presence of PDGF and bFGF and were then induced to differentiate for another $2 \mathrm{~d}$. The morphology of 
live oligodendrocytes was examined using confocal microscopy. As depicted in Figure 2, when OPCs were infected with adenovirus expressing wild-type FynEGFP, the cells extended more processes that were highly branched compared with control cells expressing EGFP. However, when OPCs were infected with adenovirus expressing (G2A)FynEGFP, morphologic differentiation of OPC was significantly inhibited, suggesting that fatty acylation of Fyn is required for inducing changes in oligodendrocyte morphology. When OPCs were infected with adenovirus expressing (K299M)FynEGFP, differentiation of OPCs was also inhibited. These results are consistent with previous studies showing a requirement for Fyn (Osterhout et al., 1999; Sperber et al., 2001) and indicate that both membrane localization and kinase activity of Fyn are essential for OPC differentiation.

\section{Function of p190RhoGAP in OPC} morphologic differentiation

We recently identified p190RhoGAP as a substrate of Fyn that becomes tyrosine phosphorylated after OPC differentiation (Wolf et al., 2001). To study the function of p190RhoGAP in oligodendrocyte differentiation, adenoviruses were generated that express HA-tagged wild-type p190RhoGAP or a mutant that is defective in GAP activity, (R1283A)p190RhoGAP. OPCs were infected overnight with adenoviruses and were induced to differentiate for $2 \mathrm{~d}$ in defined medium. The cells were then fixed, stained with anti-HA antibody, and counterstained with Alexa Red-conjugated goat anti-mouse secondary antibody. We have previously shown that p190RhoGAP colocalizes extensively with actin filaments (Wolf et al., 2001). Thus, the HA staining pattern fully elaborates the cell processes and is an accurate representation of cell morphology. To quantitate the effects of the wild-type and (R1283A)p190RhoGAP mutant on OPC differentiation, $>60$ infected cells were chosen blindly, analyzed by confocal microscopy, and divided into five categories according to their complexity: very low, low, medium, high, and very high. As depicted in Figure 3, expression of wild-type p190RhoGAP induced a more complex morphology, with a greater percentage of cells exhibiting longer and more highly branched processes. In contrast, cells expressing the (R1283A)p190RhoGAP mutant had shorter and less branched processes compared with control cells. It is likely that R1283Ap190RhoGAP acts as a dominant-negative mutant, by sequestering Rho-GTP or other essential signaling components. These results suggest that p190RhoGAP promotes OPC morphologic differentiation and that the GAP activity of p190RhoGAP is necessary for this effect.

\section{Regulation of Rho activity during} oligodendrocyte differentiation

The primary function of P190RhoGAP is to activate the GTPase activity of Rho, thereby promoting formation of Rho-GDP and Rho inactivation. To determine Rho activity during OPC differentiation, a Rhotekin-GST fusion was used to pull down activated Rho (Manser et al., 1994). Freshly prepared cell lysates from progenitor cells or differentiated oligodendrocytes were normalized to the same amount of total protein and were incubated with Sepharose beads conjugated to the Rhotekin-GST fusion. The amount of RhoA bound to Rhotekin as well as the amount of total RhoA in the whole-cell lysate was analyzed by Western blotting using anti-RhoA antibody (Fig. 4A). Levels of total RhoA protein were reduced twofold to threefold, and levels of Rho-GTP bound to Rhotekin-GST were reduced fourfold to fivefold in differentiated cells. Thus, downregulation of both RhoA protein and activity occurs during OPC differentiation.

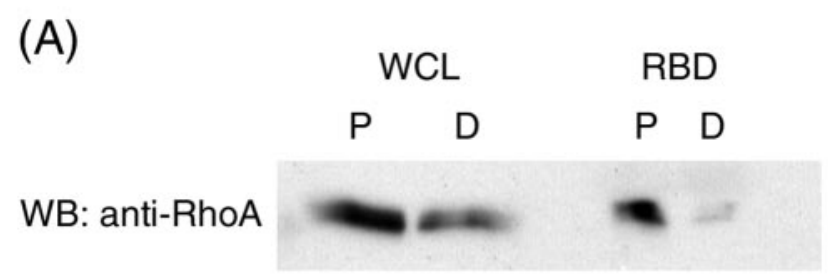

(B)

\section{WCL RBD C Wt R1283A C Wt R1283A} WB: anti-RhoA

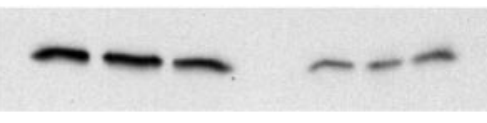

WB: anti-HA

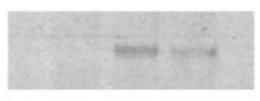

(C)

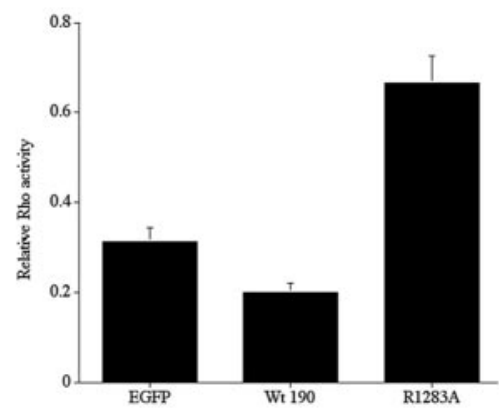

Figure 4. Regulation of RhoA activities during OPC differentiation. $A$, Cell lysates from progenitors $(P)$ and differentiated oligodendrocytes (D) grown on PDL-coated dishes were incubated with a GST fusion of the Rhotekin-binding domain (RBD) to pulldown the GTP-bound form of RhoA. The amount of RhoA in the pulldown assay as well as in the whole-cell lysate (WCL) was determined by Western blotting with anti-RhoA antibody. B, C, Quantitation of the effect of p190RhoGAP on RhoA activity. OPCs were infected overnight with adenoviruses expressing EGFP, p190RhoGAP, or (R1283A)p190RhoGAP and were then induced to differentiate for $2 \mathrm{~d}$ in differentiation medium. Cells were lysed, and RhoA activity was determined as described above. The activity of RhoA was normalized to the amount of total RhoA protein in the whole-cell lysate.

We next determined the effect of expressing p190RhoGAP on RhoA activity. OPCs were infected overnight with adenoviruses expressing EGFP, 190RhoGAP, or the GAP-defective mutant (R1283A)p190RhoGAP and were induced to differentiate for $2 \mathrm{~d}$. The amount of active RhoA was normalized to the amount of total RhoA in each sample. As depicted in Figure 4, $B$ and $C$, expression of wild-type 190RhoGAP resulted in a decrease in RhoA-specific activity. In contrast, expression of the GAP mutant caused RhoA activity to increase more than twofold. Little change in total RhoA protein levels was noted. These results indicate that exogenous expression of 190RhoGAP can regulate RhoA-specific activity.

\section{Rho family GTPases regulate OPC} morphologic differentiation

Rho family GTPases regulate the polymerization of actin and thus control cellular cytoskeletal structure. We previously 

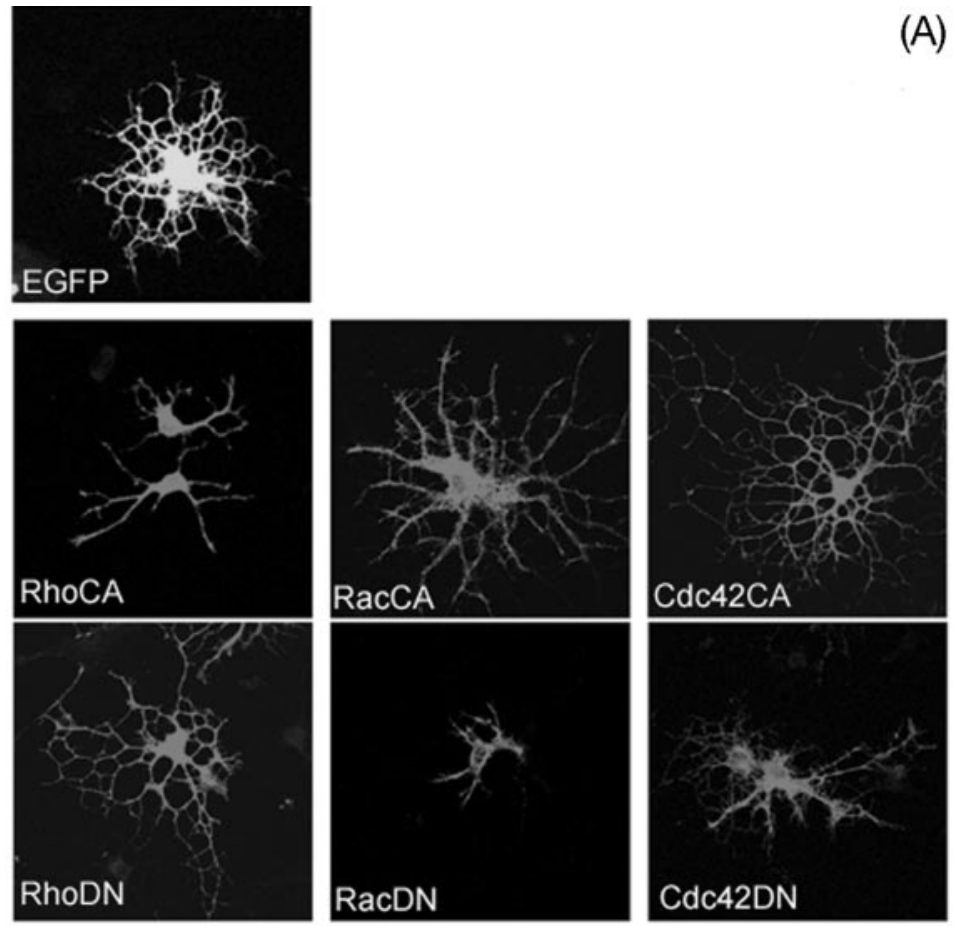

(B)
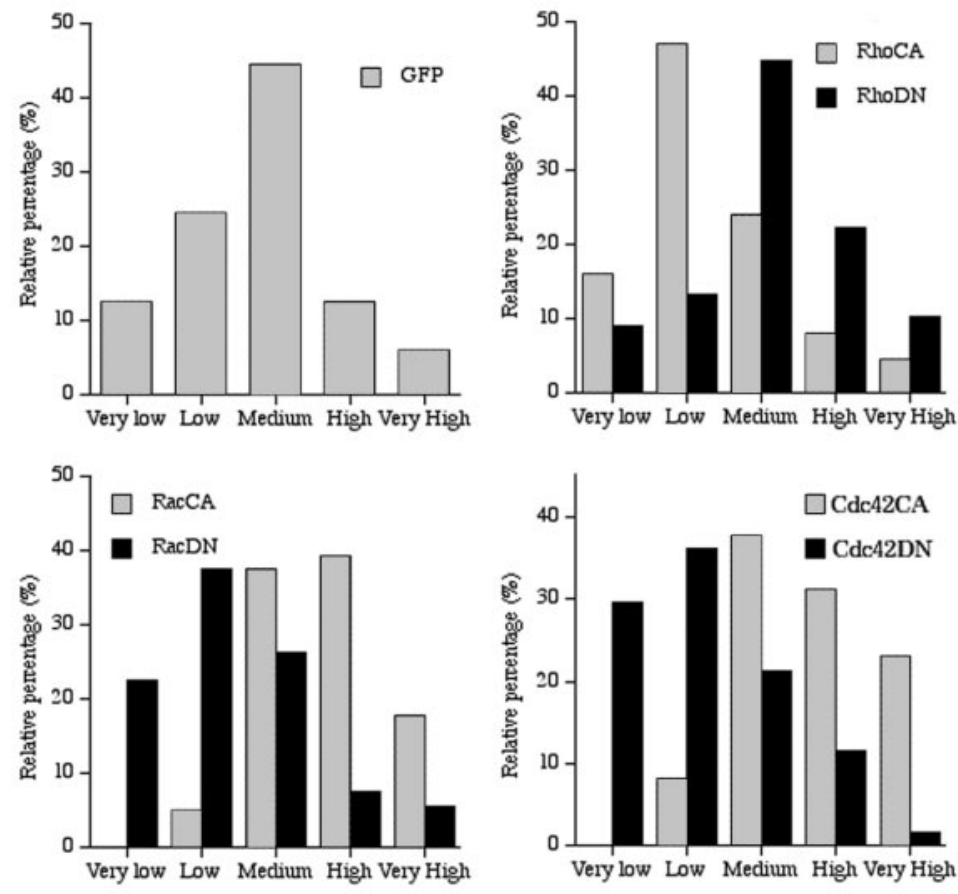

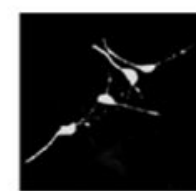

Very Low

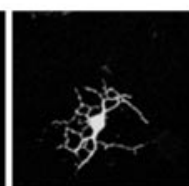

Low

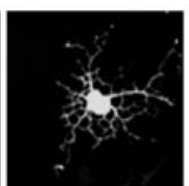

Medium

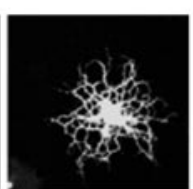

High

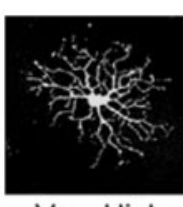

Very High
Figure 5. Constitutively active Cdc42 and Rac1 induce outgrowth of oligodendrocytes, whereas dominant-negative Cdc42 and Rac1 inhibit OPC differentiation. OPCs were infected overnight with adenoviruses expressing EGFP, CA and DN forms of RhoA, Rac1, or Cdc42, grown on PDL-coated dishes, and were then induced to differentiate for $2 \mathrm{~d}$ in defined differentiation medium. Cells were stained with anti-HA antibody and counterstained with Alexa Red-conjugated goat anti-mouse antibody. A, Representative morphology of oligodendrocytes expressing EGFP, or constitutively active and dominant-negative RhoA, Rac1, or Cdc42. B, Quantitation of the morphology of oligodendrocytes according to its complexity as described in Figure $3 B$. Results were subjected to a paired $t$ test. The differences between wt and mutant constructs were deemed statistically significant: $p<0.01$ for RhoA, $p<0.005$ for Rac1, and $p<0.02$ for Cdc42. showed that expression of constitutively active RhoA inhibited process extension in oligodendrocytes, whereas a dominantnegative mutant of RhoA induced hyperextension of oligodendrocyte processes (Wolf et al., 2001). To determine the contribution of the other Rho family members, Cdc42 and Rac, on morphologic differentiation of oligodendrocytes, adenoviruses were generated that express constitutively activated and dominant-negative HA-tagged RhoA, Rac1, or Cdc42. Oligodendrocyte progenitor cells were infected overnight and were then induced to differentiate for $2 \mathrm{~d}$ in defined medium. Cells were fixed and immunostained with anti-HA antibody and counterstained with Alexa Red-conjugated goat antimouse secondary antibody. Cell morphology was examined using confocal microscopy. To quantitate the effect of Rho family GTPases on OPC differentiation, we blindly selected infected cells and divided them into five categories according to their complexity, as described above. As depicted in Figure 5, $A$ and $B$, expression of dominant-negative RhoA increased the percentage of oligodendrocytes with highly branched, hyperextended processes. In contrast, expression of constitutively active Cdc42 or Racl caused hyperextension of oligodendrocyte processes, whereas expression of dominant-negative Cdc42 or Racl inhibited morphologic differentiation of oligodendrocytes. These data are consistent with the notion that Rho and Cdc42-Rac are reciprocally regulated and exhibit opposite effects on process extension.

\section{Regulation of Cdc42 and Rac1 during oligodendrocyte differentiation} To determine whether Cdc42 and/or Rac1 were being activated during oligodendrocyte differentiation, the activities of $\mathrm{Cdc} 42$ and Racl were measured biochemically. Cdc42 and Rac1 in their GTP-bound states interact specifically with PAK, p21activated kinase (Manser et al., 1994). A GST-fusion protein containing the PBD was incubated with cell lysates from progenitors and differentiated oligodendrocytes, and the amounts of Cdc42 and Rac1 bound to the GST fusion protein, as well as the amount of total Cdc42 and Racl in the whole-cell lysate, were analyzed by Western blotting. As depicted in Figure 6 A, both the activities as well as the expression levels of Cdc42 and Racl increased after differentiation of oligodendrocytes, with $\sim 2.5$-fold and 2.3-fold increases in specific activity for Cdc42 and Rac1, respectively.

To establish the links between integrin, Fyn, and Cdc42-Racl during differentia- 
tion, OPCs were preincubated for $1 \mathrm{hr}$ with control serum or anti- $\beta 1$ antibody and were then plated on $\mathrm{FN}$-coated dishes for $5 \mathrm{~d}$ in differentiation medium in the presence of control serum or anti-integrin $\beta 1$ antibody. The activities of Cdc42 and Racl were then determined by the PAKGST pulldown assay. As depicted in Figures $1 C$ and $6 B$, disruption of extracellular matrix interaction by anti- $\beta 1$ antibody blocked activation of Cdc42 and Rac1 as well as OPC morphologic differentiation and expression of MBP. To determine whether Fyn was responsible for activation of Cdc42 and/or Rac1, OPCs were induced to differentiate for $2 \mathrm{~d}$ in the presence of the Src family kinase inhibitor PP2 $(3 \mu \mathrm{M})$ or the nonfunctional analog PP3, and the activities of Rac1 and Cdc42 were measured. As depicted in Figure $6 B$, treatment of OPC with PP2 inhibited activation of Rac1 and Cdc42 as well as morphologic differentiation of OPC and expression of MBP. As a control, PP2 had little effect on the amount of p190 in the cells. These data imply that integrin and Fyn activation is upstream of Cdc42-Rac1.

We next addressed the potential cross talk between and among p190, Rho, and Rac1. Purified p190 has been reported to function in vitro as a GAP for both RhoA and Rac1 (Settleman et al., 1992; Ligeti et al., 2004). To test whether this can occur in vivo, COS-1 cells were cotransfected with p190 (wild-type or R1283A mutant) and either HA-tagged Rac1 or Cdc42. As de-

picted in Figure 7A, wild-type, but not GAP-defective p190 was able to decrease the amount of active Rac1. No change in Rac1 total protein levels was observed. Moreover, there was no effect of p190 expression on levels of total or active Cdc42.

To examine cross talk between Rho and Rac1-Cdc42, we performed Rhotekin pulldown assays on COS-1 cell lysates that had been transfected with dominant-negative or constitutively activated Rac1 or Cdc42. No apparent changes in either the amount of total RhoA or activated RhoA (RhoGTP) were observed (Fig. $7 B$ ). Finally, we used a differentiated oligodendrocyte cell line, O2Amyc (Barnett and Crouch, 1995), to examine whether Fyn was able to regulate Rho activity. As depicted in Figure $7 C$, an increase in activated Rho was observed when cells were pretreated with the Src family kinase inhibitor PP2. This is consistent with the ability of Fyn to upregulate p190 (Wolf et al., 2001). Taken together, these data suggest a signal transduction pathway of integrin-Fyn-Rho family GTPases that controls oligodendrocyte morphology and myelination.

\section{Discussion}

In this study, we have identified signaling components that operate upstream and downstream of Fyn to trigger alterations in oligodendrocyte morphology during differentiation. There is extensive evidence in the literature to indicate a central role for Fyn in myelination and oligodendrocyte differentiation (Umemori et al., 1999; Sperber et al., 2001), but few studies have examined Fyn targets in oligodendrocytes. Here we show that integrin engage-

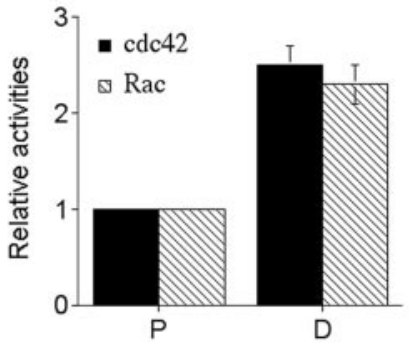

\section{WCL \\ C anti- $\beta 1 \quad C$ anti- $\beta 1$}
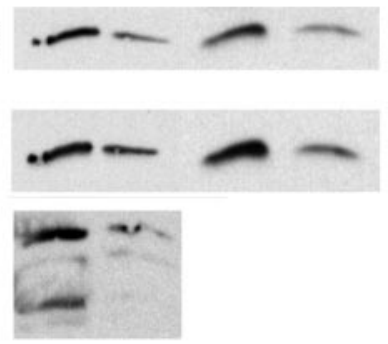
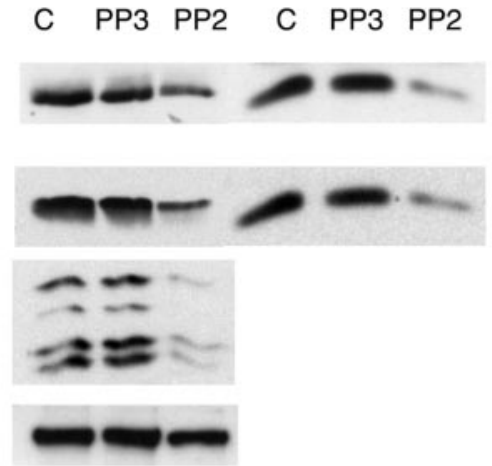

Figure 6. Regulation of $\mathrm{Cd} 422$ and Rac1 activities during $0 \mathrm{PC}$ differentiation. $A$, Cell lysates from progenitors (P) and differenoligodendrocytes (D) grown on PDL-coated dishes were incubated with a GST fusion containing the PAK-binding domain

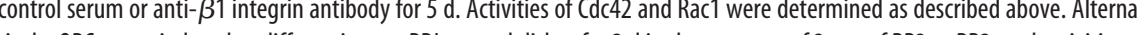
OPCs were induced to differentiate on PDL-coated dishes for $2 d$ in the presence of $3 \mu \mathrm{m}$ of PP3 or PP2, and activities of Cdc42-Rac1 were determined. Expression of MBP and p190 in whole-cell lysates was determined by Western blotting with anti-MBP and anti-p190 antibodies, respectively.

ment activates Fyn in primary oligodendrocytes. Moreover, treatment of progenitor cells with an anti- $\beta 1$ integrin blocking antibody inhibited both Fyn activation and morphologic differentiation. These findings are consistent with previous studies demonstrating that $\beta 1$ integrin function is important for myelination in oligodendrocytes and Schwann cells (Buttery and ffrench-Constant, 1999; Relvas et al., 2001; Feltri et al., 2002). In addition, a link between integrins and Src family kinases, especially Fyn, is well established in fibroblasts and endothelial cells (Wary et al., 1998; Felsenfeld et al., 1999). The anti-integrin $\beta 1$ antibody was present in the media for $5 \mathrm{~d}$, the time required for full morphological differentiation of oligodendrocytes. Thus, multiple events could have occurred during the course of the treatment. However, because upregulation of Fyn is a very early event in oligodendrocyte differentiation (Osterhout et al., 1999), it is likely that effects of blocking integrin signaling also occur early during the differentiation process. It should be noted that other processes, besides integrin engagement, likely also contribute to Fyn activation in oligodendrocytes, such as Ig Fc receptor engagement (Nakahara et al., 2003) and association with GPIanchored proteins in rafts (Kramer et al., 1999).

Several recent studies have identified downstream targets for Fyn in oligodendrocytes. These include the F3 adhesion molecule, the cytoskeletal proteins tau and $\alpha$-tubulin, and two proteins that regulate Rho activity, p190RhoGAP and p250RhoGAP (Wolf et al., 2001; Klein et al., 2002; Taniguchi et al., 2003). Our laboratory previously demonstrated that Fyn phosphorylates 
(A)

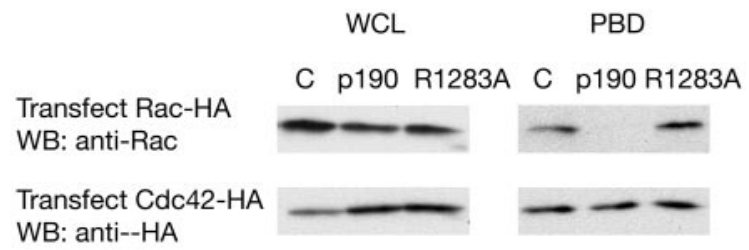

(B)

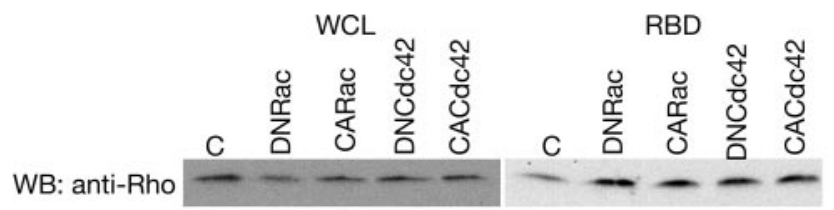

(C)

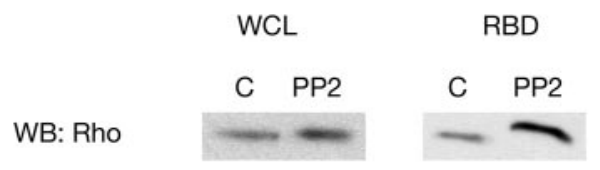

Figure 7. Cross talk among p190, Rho, Rac1, and Cdc42. A, COS-1 cells were cotransfected with empty vector (C), wt p190, or GAP-defective p190 (R1283A) and either HA-tagged Rac1 or Cdc42. Activities of Rac1 and Cdc42 were determined as described in Figure 6. B, COS-1 cells were transfected with DN or CA forms of Rac1 or $\mathrm{Cdc} 42$, and Rho activity was determined as described in Figure 4. C, Differentiated 02Amyc oligodendrocytes grown on PDL-coated dishes were treated with buffer or $3 \mu \mathrm{MPP} 2$, as described in Figure 6, and Rho activity was determined.

p190RhoGAP during oligodendrocyte differentiation and thereby increases p190 GAP activity toward Rho (Wolf et al., 2001). We postulated that $\mathrm{p} 190$-induced generation of Rho-GDP was essential for regulating process extension during differentiation. This hypothesis was supported by our finding that transfection of primary oligodendrocytes with dominant-negative (DN) Rho, which mimics Rho in the GDP-bound state, was sufficient to promote hyperextension of cellular processes.

A major limitation of the use of primary oligodendrocytes has been the low transfection efficiency that can be achieved with these cells. We, therefore, exploited recombinant adenovirus technology to efficiently express heterologous proteins in progenitor cells and determine their effects on oligodendrocyte morphology during differentiation. In addition, we adopted an oligodendrocyte complexity scoring system (Sperber and McMorris, 2001; Sperber et al., 2001) to quantitate the extent of process extension and cell arborization in cells infected with the various recombinant adenoviruses. Our results establish that expression of wt p190RhoGAP increases the percentage of more morphologically complex cells. In contrast, expression of the R1283A GAPdefective p190 mutant inhibited these changes, with $\sim 60 \%$ of the cells exhibiting very low or low complexity, compared with $35 \%$ of the GFP control and $<10 \%$ of the wt p190-expressing cells. These data imply that GAP activity of p190 is important for its function in inducing alterations in oligodendrocyte morphology.

The scoring system that we used was designed to evaluate morphological complexity, a parameter that we and others have equated with differentiation. However, it is also possible that changes in morphology are reflecting alterations in cytoskeletal organization or myelin membrane elaboration, rather than the differentiation process per se. Although we did observe a decrease in the overall amount of MBP when cells were treated with antiintegrin $\beta 1$ antibody or with PP2, quantitation of MBP levels in cellular processes has not been performed. Moreover, it is not clear whether the reduction in the number and complexity of processes that was observed in this study correlates directly with the reduction in the number of myelin lamellae observed in studies of Fyn $-/-$ mice.

The presence of p190 is essential for normal neural development. The p190 knock-out mouse exhibits an embryonic lethal phenotype, with defects in neural tube closure, axon guidance, corpus callosum formation, and eye development (Brouns et al., 2001). p190 is the major tyrosine-phosphorylated protein in the brain, and Src and Fyn are the kinases primarily responsible for p190 phosphorylation (Brouns et al., 2000). Although glial cell morphology was not directly evaluated in the p190-/- mice, our findings suggest that defects in oligodendrocyte differentiation should be present.

Overexpression of $\mathrm{p} 190$ likely functions by promoting formation of Rho-GDP and thereby downregulating Rho signaling. Indeed, levels of Rho-GTP were decreased in differentiated oligodendrocytes, compared with progenitors (Fig. 4A). Rho-GTP levels were modestly decreased in cells expressing wt p190, compared with control cells (Fig. $4 B$ ). It is possible that other GAPs, or other regulatory proteins, are involved in the downregulation of Rho activity during differentiation. For example, a recent report identified another Rho GAP, p250, as a Fyn substrate in differentiated oligodendrocytes (Taniguchi et al., 2003). The mechanism whereby total levels of RhoA are downregulated during differentiation is unknown. However, it does not appear to be mediated by p190, because little to no change in RhoA protein levels was observed in cells overexpressing wild-type or mutant p190 constructs. Interestingly, overexpression of the GAPdefective p190 mutant resulted in a twofold to threefold increase in Rho-GTP levels. The R1283A p190 may function as a dominant-negative mutant, by binding essential factors through its other domains.

p190 has been shown to exhibit GAP activity toward Racl as well as RhoA, particularly in vitro (Settleman et al., 1992; Ridley et al., 1993; Ligeti et al., 2004). Indeed, when p190 was overexpressed in COS- 1 cells, the amount of activated Racl was reduced (Fig. 7A). However, this level of overexpression is unlikely to occur under physiologic conditions in oligodendrocytes, and other studies have shown that p190 exhibits a preference for regulating Rho over Rac1 in vivo (Ridley et al., 1993). Our data reveal that Rac1 and Cdc42 are activated in differentiated cells, a finding that cannot be caused by increased p190RhoGAP activity. Moreover, constitutively activated (CA) Rac and Cdc42 promoted the formation of more highly differentiated cells with higher levels of complexity, whereas DN versions of these proteins had the opposite effect. These results imply that Rho and Cdc42-Rac play opposing roles in oligodendrocyte morphologic differentiation. A similar situation has been observed in neurons, where Rac and Cdc42 are positive regulators that promote neurite extension, whereas RhoA mediates neurite retraction (Jalink et al., 1994; Kozma et al., 1997; Gallo and Letourneau, 1998; Katoh et al., 2000).

Cross talk between Cdc42-Rac and Rho has been observed in several other systems. For example, fibroblasts have a linear pathway in which activation of Cdc42 leads to activation of Racl and then Rho resulting in generation of filopodia (Nobes and Hall, 1995). In neuronal cells and epithelial cells, Rac activation downregulates Rho activity (Kozma et al., 1997; Sander et al., 1999; 
Tashiro et al., 2000; Wong et al., 2000; Li et al., 2002). The balance and reciprocal regulation of Rho versus Rac-Cdc42 is likely to be achieved by multiple factors, including GAPs and GEFs. Our study is the first to establish a sequential signaling pathway, from integrins to Fyn to Rho family proteins, that results in oligodendrocyte maturation and process extension (supplemental Fig. 1, available at www.jneurosci.org/cgi/content/full/24/32/7140/ DC1). We propose that Fyn functions as one of the factors that reciprocally regulates Rho and Rac-Cdc42. Phosphorylation of p190 by Fyn promotes increased p190 Rho GAP activity, leading to a decrease in activated Rho, whereas at the same time, Fyn activation leads to Rac-Cdc42 activation. Additional experiments will be needed to fully elucidate all the factors that regulate cross talk between RhoA and Rac1-Cdc42. Knowledge of the pathways that promote oligodendrocyte differentiation is important furthering our understanding of the mechanisms regulating myelination in the CNS.

\section{References}

Arthur WT, Burridge K (2001) RhoA inactivation by p190RhoGAP regulates cell spreading and migration by promoting membrane protrusion and polarity. Mol Biol Cell 12:2711-2720.

Barnett SC, Crouch DH (1995) The effect of oncogenes on the growth and differentiation of oligodendrocyte type 2 astrocyte progenitor cells. Cell Growth Differ 6:69-80.

Berrier AL, Martinez R, Bokoch GM, LaFlamme SE (2002) The integrin beta tail is required and sufficient to regulate adhesion signaling to Rac1. J Cell Sci 115:4285-4291.

Blaschuk KL, Frost EE, ffrench-Constant C (2000) The regulation of proliferation and differentiation in oligodendrocyte progenitor cells by alphaV integrins. Development 127:1961-1969.

Brouns MR, Matheson SF, Hu KQ, Delalle I, Caviness VS, Silver J, Bronson RT, Settleman J (2000) The adhesion signaling molecule p190 RhoGAP is required for morphogenetic processes in neural development. Development 127:4891-4903.

Brouns MR, Matheson SF, Settleman J (2001) p190 RhoGAP is the principal Src substrate in brain and regulates axon outgrowth, guidance and fasciculation. Nat Cell Biol 3:361-367.

Buttery PC, ffrench-Constant C (1999) Laminin-2/integrin interactions enhance myelin membrane formation by oligodendrocytes. Mol Cell Neurosci 14:199-212.

Cheresh DA, Leng J, Klemke RL (1999) Regulation of cell contraction and membrane ruffling by distinct signals in migratory cells. J Cell Biol 146:1107-1116.

Das B, Shu X, Day GJ, Han J, Krishna UM, Falck JR, Broek D (2000) Control of intramolecular interactions between the pleckstrin homology and $\mathrm{Dbl}$ homology domains of Vav and Sos1 regulates Rac binding. J Biol Chem 275:15074-15081.

del Pozo MA, Price LS, Alderson NB, Ren XD, Schwartz MA (2000) Adhesion to the extracellular matrix regulates the coupling of the small GTPase Rac to its effector PAK. EMBO J 19:2008-2014.

Felsenfeld DP, Schwartzberg PL, Venegas A, Tse R, Sheetz MP (1999) Selective regulation of integrin-cytoskeleton interactions by the tyrosine kinase Src. Nat Cell Biol 1:200-206.

Feltri ML, Graus Porta D, Previtali SC, Nodari A, Migliavacca B, Cassetti A, Littlewood-Evans A, Reichardt LF, Messing A, Quattrini A, Mueller U, Wrabetz L (2002) Conditional disruption of beta 1 integrin in Schwann cells impedes interactions with axons. J Cell Biol 156:199-209.

Gallo G, Letourneau PC (1998) Axon guidance: GTPases help axons reach their targets. Curr Biol 8:R80-R82.

Jalink K, van Corven EJ, Hengeveld T, Morii N, Narumiya S, Moolenaar WH (1994) Inhibition of lysophosphatidate- and thrombin-induced neurite retraction and neuronal cell rounding by ADP ribosylation of the small GTP-binding protein Rho. J Cell Biol 126:801-810.

Katoh H, Yasui H, Yamaguchi Y, Aoki J, Fujita H, Mori K, Negishi M (2000) Small GTPase RhoG is a key regulator for neurite outgrowth in PC12 cells. Mol Cell Biol 20:7378-7387.

Klein C, Kramer EM, Cardine AM, Schraven B, Brandt R, Trotter J (2002)
Process outgrowth of oligodendrocytes is promoted by interaction of fyn kinase with the cytoskeletal protein tau. J Neurosci 22:698-707.

Klemke RL, Leng J, Molander R, Brooks PC, Vuori K, Cheresh DA (1998) CAS/Crk coupling serves as a "molecular switch" for induction of cell migration. J Cell Biol 140:961-972.

Kozma R, Ahmed S, Best A, Lim L (1995) The Ras-related protein Cdc42Hs and bradykinin promote formation of peripheral actin microspikes and filopodia in Swiss 3T3 fibroblasts. Mol Cell Biol 15:1942-1952.

Kozma R, Sarner S, Ahmed S, Lim L (1997) Rho family GTPases and neuronal growth cone remodelling: relationship between increased complexity induced by $\mathrm{Cdc} 42 \mathrm{Hs}$, Racl, and acetylcholine and collapse induced by RhoA and lysophosphatidic acid. Mol Cell Biol 17:1201-1211.

Kramer EM, Klein C, Koch T, Boytinck M, Trotter J (1999) Compartmentation of Fyn kinase with glycosylphosphatidylinositol-anchored molecules in oligodendrocytes facilitates kinase activation during myelination. J Biol Chem 274:29042-29049.

Liang X, Nazarian A, Erdjument-Bromage H, Bornmann W, Tempst P, Resh MD (2001) Heterogeneous fatty acylation of Src family kinases with polyunsaturated fatty acids regulates raft localization and signal transduction. J Biol Chem 276:30987-30994.

Li Z, Aizenman CD, Cline HT (2002) Regulation of rho GTPases by cross talk and neuronal activity in vivo. Neuron 33:741-750.

Ligeti E, Dagher M-C, Hernande SE, Koleske AJ, Settleman J (2004) Phospholipids can switch the GTPase substrate preference of a GTPase activating protein. J Biol Chem 279:5055-5058.

Malek-Hedayat S, Rome LH (1994) Expression of a beta 1-related integrin by oligodendroglia in primary culture: evidence for a functional role in myelination. J Cell Biol 124:1039-1046.

Manser E, Leung T, Salihuddin H, Zhao ZS, Lim L (1994) A brain serine/ threonine protein kinase activated by $\mathrm{Cdc} 42$ and Racl. Nature $367: 40-46$

Milner R, Ffrench-Constant C (1994) A Developmental analysis of oligodendroglial integrins in primary cells: changes in $\alpha \mathrm{v}$-associated $\beta$ subunits during differentiation. Development 120:3497-3506.

Nakahara J, Tan-Takeuchi K, Seiwa C, Gotoh M, Kaifu T, Ujike A, Inui M, Yagi T, Ogawa M, Aiso S, Takai T, Asou H (2003) Signaling via immunoglobulin Fc receptors induces oligodendrocyte precursor cell differentiation. Dev Cell 4:841-852.

Nobes CD, Hall A (1995) Rho, rac, and cdc42 GTPases regulate the assembly of multimolecular focal complexes associated with actin stress fibers, lamellipodia, and filopodia. Cell 81:53-62.

Osterhout DJ, Wolven A, Wolf RM, Resh MD, Chao MV (1999) Morphological differentiation of oligodendrocytes requires activation of Fyn tyrosine kinase. J Cell Biol 145:1209-1218.

Price LS, Leng J, Schwartz MA, Bokoch GM (1998) Activation of Rac and Cdc42 by integrins mediates cell spreading. Mol Biol Cell 9:1863-1871.

Relvas JB, Setzu A, Baron W, Buttery PC, LaFlamme SE, Franklin RJ, ffrenchConstant C (2001) Expression of dominant-negative and chimeric subunits reveals an essential role for betal integrin during myelination. Curr Biol 11:1039-1043

Ren XD, Kiosses WB, Schwartz MA (1999) Regulation of the small GTPbinding protein Rho by cell adhesion and the cytoskeleton. EMBO J 18:578-585.

Ridley AJ, Paterson HF, Johnston CL, Diekmann D, Hall A (1992) The small GTP-binding protein rac regulates growth factor-induced membrane ruffling. Cell 70:401-410.

Ridley AJ, Self AJ, Kasmi F, Paterson HF, Hall A, Marshall CJ, Ellis C (1993) rho family GTPase activating proteins p190, bcr and rhoGAP show distinct specificities in vitro and in vivo. EMBO J 12:5151-5160.

Sander EE, ten Klooster JP, van Delft S, van der Kammen RA, Collard JG (1999) Rac downregulates Rho activity: reciprocal balance between both GTPases determines cellular morphology and migratory behavior. J Cell Biol 147:1009-1022.

Schaller MD, Hildebrand JD, Parsons JT (1999) Complex formation with focal adhesion kinase: a mechanism to regulate activity and subcellular localization of Src kinases. Mol Biol Cell 10:3489-3505.

Schlaepfer DD, Broome MA, Hunter T (1997) Fibronectin-stimulated signaling from a focal adhesion kinase-c-Src complex: involvement of the Grb2, p130cas, and Nck adaptor proteins. Mol Cell Biol 17:1702-1713.

Schwartz MA (2001) Integrin signaling revisited. Trends Cell Biol $11: 466-470$. 
Settleman J, Albright CF, Foster LC, Weinberg RA (1992) Association between GTPase activators for Rho and Ras families. Nature 359:153-154.

Sperber BR, McMorris FA (2001) Fyn tyrosine kinase regulates oligodendroglial cell development but is not required for morphological differentiation of oligodendrocytes. J Neurosci Res 63:303-312.

Sperber BR, Boyle-Walsh EA, Engleka MJ, Gadue P, Peterson AC, Stein PL, Scherer SS, McMorris FA (2001) A unique role for Fyn in CNS myelination. J Neurosci 21:2039-2047.

Taniguchi S, Liu H, Nakazawa T, Yokoyama K, Tezuka T, Yamamoto T (2003) p250GAP, a neural RhoGAP protein, is associated with and phosphorylated by Fyn. Biochem Biophys Res Commun 306:151-155.

Tashiro A, Minden A, Yuste R (2000) Regulation of dendritic spine morphology by the rho family of small GTPases: antagonistic roles of Rac and Rho. Cereb Cortex 10:927-938.
Umemori H, Kadowaki Y, Hirosawa K, Yoshida Y, Hironaka K, Okano H, Yamamoto T (1999) Stimulation of myelin basic protein gene transcription by Fyn tyrosine kinase for myelination. J Neurosci 19:1393-1397.

van't Hof W, Resh MD (1997) Rapid plasma membrane anchoring of newly synthesized p59fyn: selective requirement for NH2-terminal myristoylation and palmitoylation at cysteine-3. J Cell Biol 136:1023-1035.

Wary KK, Mariotti A, Zurzolo C, Giancotti FG (1998) A requirement for caveolin-1 and associated kinase Fyn in integrin signaling and anchoragedependent cell growth. Cell 94:625-634.

Wolf RM, Wilkes JJ, Chao MV, Resh MD (2001) Tyrosine phosphorylation of p190 RhoGAP by Fyn regulates oligodendrocyte differentiation. J Neurobiol 49:62-78.

Wong WT, Faulkner-Jones BE, Sanes JR, Wong RO (2000) Rapid dendritic remodeling in the developing retina: dependence on neurotransmission and reciprocal regulation by Rac and Rho. J Neurosci 20:5024-5036. 